
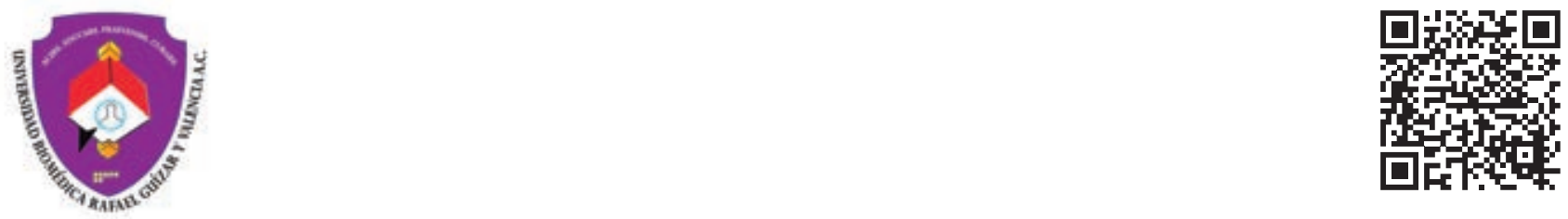

Vol. 8, Núm. 1

Enero-Junio 2021. pp. 50-53

doi: 10.35366/101205

Nuevos horizontes en la restauración neurológica

\title{
Deficiencia de vitamina D. Conceptos actuales
}

\author{
Vitamin D deficiency. Current concepts
}

\author{
Daniel Sánchez Ruiz, * Francisco Aguilar Rebolledo
}

\section{RESUMEN}

Antecedentes: La vitamina D es una hormona liposoluble que existe en dos formas moleculares. Ergocalciferol (vitamina D-2) y colecalciferol (vitamina D-3). La vitamina D-3 se produce en la piel por la acción de la radiación UVB. Ambas formas son metabolizadas por el hígado a 25-hidroxi-Vit D (25OHD) y luego en el riñón a la forma activa 1,25-dihidroxi-Vit D. Esta forma promueve la mineralización ósea mediante la absorción intestinal de calcio y fosfato. Los niveles normales de 25OHD se asocian con menos fracturas, función neuromuscular e inmunológica normal y posiblemente tengan un efecto preventivo sobre ciertos tipos de cáncer. Recomienda que los niveles plasmáticos óptimos de 25OHD estén por encima de $30 \mathrm{ng} / \mathrm{mL}$, la insuficiencia entre 21 y $29 \mathrm{ng} / \mathrm{mL}$ y la deficiencia por debajo de 20 $\mathrm{ng} / \mathrm{mL}$. Resultados: La tasa de prevalencia del déficit de 25OHD es de aproximadamente 2 a $90 \%$ en diferentes poblaciones. Los factores de riesgo del déficit de vitamina $\mathrm{D}$ como la estación del año, la pigmentación de la piel, la exposición al sol, el uso de bloqueador solar y la ingesta inadecuada de vitamina $\mathrm{D}$, junto con las diferentes técnicas de medición, explican la variabilidad de los resultados entre los estudios epidemiológicos. Un grupo de riesgo importante son los profesionales de la salud que no están expuestos a la luz solar. Un estado bajo de vitamina $\mathrm{D}$, medido como el nivel plasmático de la forma de transporte de vitamina $\mathrm{D}, 25(\mathrm{OH}) \mathrm{D}$.

Palabras clave: Vitamina D, deficiencia, acciones en hueso, sistema inmune.

\begin{abstract}
Background: Vitamin D is a liposoluble hormone that exists in two molecular forms. Ergocalciferol (vitamin D-2) and colecalciferol (vitamin D-3). Vitamin D-3 is produced in the skin by the action of $U V-B$ radiation. Both forms are metabolized by the liver to 25-hydroxy-Vit D (25OHD) and later in the kidney to the active form 1,25-dihydroxy-Vit $D$. This form promotes bone mineralization by intestinal absorption of calcium and phosphate. Normal levels of $250 H D$ are associated with less fracture, normal neuromuscular and immune function and possibly have a preventive effect on certain types of cancer. Recommends that optimal plasma levels of $25 O H D$ are above $30 \mathrm{ng} / \mathrm{mL}$, insufficiency between 21 and $29 \mathrm{ng} /$ $m L$ and deficiency below $20 \mathrm{ng} / \mathrm{mL}$. Results: The prevalence rate of $25 \mathrm{OHD}$ deficit is about 2 to $90 \%$ in different populations. Risk factors of Vitamin D deficit like year season, skin pigmentation, sunlight exposition, use of sunblock and inadecuate Vitamin D ingestion, together with different measurement techniques explain the variability of results between epidemiological studies. An important risk group is the health professionals that are not exposed to sunlight. A low vitamin D status, measured as the plasma level of the transport form of vitamin $D, 25(\mathrm{OH}) \mathrm{D}$.
\end{abstract}

Keywords: Vitamin D, deficiency, actions in bone, immune system.

* Coordinador de la Licenciatura de Nutrición UNIBIO.

* Rector de la Universidad Biomédica «Rafael Guízar y Valencia».

\author{
Correspondencia: \\ Daniel Sánchez Ruíz \\ E-mail: nutricion@universidadbiomedicargyv.com
}

Recibido: 17-07-2021. Aceptado: 31-07-2021.

Citar como: Sánchez RD, Aguilar RF. Deficiencia de vitamina D. Conceptos actuales.

Plast Restaur Neurol. 2021;8 (1): 50-53. https://dx.doi.org/10.35366/101205 


\section{INTRODUCCIÓN}

La vitamina $\mathrm{D}$ pertenece a la familia de las vitaminas liposolubles, pero es considerada una hormona dada su obtención a través de la síntesis cutánea en presencia de exposición a la luz solar. Hay dos formas moleculares con diferentes fuentes de obtención:

1. La vitamina D-2 (ergocalciferol) se encuentra en plantas y en suplementos de fortificación y no es sintetizada por el hombre.

2. La vitamina D-3 (colecalciferol), de origen animal, que proviene principalmente de la bioconversión cutánea que se genera por la radiación ultravioleta UVB.

3. También se puede ingerir a través de fuentes animales y suplementos. ${ }^{1-3}$

La síntesis cutánea representa aproximadamente $90 \%$ del total de vitamina en plasma. ${ }^{2}$ Ambas formas se hidroxilan a nivel hepático para obtener la 25-hidroxi-vitamina D (25OHD), la cual es utilizada por consenso para establecer los niveles plasmáticos de vitamina $\mathrm{D}$.

Finalmente, a través de una segunda hidroxilación enzimática a nivel renal y en varios otros tejidos, se obtiene la 1,25-dihidroxi-vitamina $D$, que es la forma biológicamente activa. ${ }^{1-3}$

La acción principal de la vitamina $\mathrm{D}$ tiene relación con el metabolismo calcio-óseo, promoviendo la mineralización del hueso a través de la absorción de calcio y fósforo a nivel intestinal y renal para mantener niveles de calcio y de hormona paratiroidea (PTH) adecuados. Clásicamente el déficit de vitamina $\mathrm{D}$ en adultos se asocia a osteomalacia y miopatía proximal por vitamina $\mathrm{D}$.

La vitamina $D$ juega un papel clave en la respuesta del huésped contra las infecciones, incluido el refuerzo de las acciones antimicrobianas y la modulación de la respuesta inflamatoria. Este último está mediado por varias vías que involucran directamente al sistema inmunológico, pero también por la regulación del sistema renina-angiotensina y de la enzima convertidora de angiotensina 2 (ACE2).

La osteomalacia se define como una alteración de la matriz ósea con un aumento de tejido no mineralizado u osteoide, lo que determina una alteración en la calidad del hueso con aumento de la hidratación del tejido óseo. En los niños, el déficit de vitamina D afecta el cartílago de crecimiento y genera signos típicos que configuran el raquitismo. Una severa y prolongada deficiencia de 25OHD (completas o pseudofracturas a nivel de pelvis, costillas y escápula). ${ }^{4}$
Los efectos del envejecimiento y el aumento de la comorbilidad asociado se traducen en una interacción compleja de los mecanismos causales de morbilidad y mortalidad en el subconjunto de pacientes de mayor edad con COVID-19 que es difícil de interpretar. Además, el envejecimiento conlleva una disfunción de las respuestas inmunitarias normales y una profunda desregulación de las vías inflamatorias.

\section{Desarrollo}

En relación a los beneficios de mantener un estatus adecuado de vitamina $D$, hay evidencia en metaanálisis que reporta asociación de los niveles plasmáticos óptimos de 25OHD (> $24 \mathrm{ng} / \mathrm{mL}$ ) con un menor riesgo de fractura de cadera, no vertebrales y de caídas, además de una adecuada función neuromuscular. ${ }^{5}$

Por otra parte, hay estudios observacionales que sugieren que la vitamina $D$ sería un potencial regulador de otras funciones celulares, confiriéndole un rol en la mantención de la inmunidad innata, en la inhibición de la proliferación celular, angiogénesis, inhibición de la síntesis de renina y estimulación de la síntesis de insulina. Se asociaría, de esta manera, a la prevención de algunas enfermedades, por ejemplo: cáncer de mama y colorrectal, patología cardiovascular, diabetes y síndrome metabólico, entre otros. Sin embargo, falta evidencia de ensayos clínicos que sustenten estos efectos, siendo actualmente objeto de continua investigación. ${ }^{5-8}$ Aproximadamente, un billón de personas tiene déficit de vitamina $\mathrm{D},{ }^{9}$ con una prevalencia que oscila entre 2 y $90 \%$, dependiendo del punto de corte utilizado y la población seleccionada. ${ }^{10}$

Se reporta una prevalencia de deficiencia $(\leq 20 \mathrm{ng} /$ $\mathrm{mL}$ ) de $37.3 \%$ e insuficiencia de vitamina D de $88.1 \%$. Hasta el 2010, la ingesta diaria recomendada (RDA) de vitamina D para adultos es:

1. De 7-dehidrocolesterol luz UV en la piel dieta

2. Suplementos colecalciferol (vitamina D3)

3. Ergocalciferol (vitamina D2)

4. Hígado calcidiol (25-hidroxivitamina D)

5. Riñón calcitriol (1,25-dihidroxivitamina D)

6. Metabolito inactivo (24,25-dihidroxivitamina D) $\downarrow$

7. Excreción renal de calcio y fósforo $\uparrow$

8. Resorción ósea $\uparrow$ Absorción intestinal de calcio

Vías de síntesis de vitamina $\mathrm{D}^{3} 400 \mathrm{UI}$; los niveles fueron aumentados a $600 \mathrm{UI}$, pero siguen estando lejos de lo necesario en grupos de alto riesgo para mantener niveles adecuados de 25OHD. En nuestro país se fortifican algunos alimentos como aceites, leches 
descremadas, mantequillas, productos de los programas de alimentación complementaria y los cereales. Así, sólo una pequeña cantidad (30\%) de vitamina D puede ser obtenida de la dieta. ${ }^{11}$ El objetivo de nuestra revisión es describir la magnitud del déficit de vitamina $D$ en América Latina incluido nuestro país y los factores de riesgo que determinan este problema mundial.

\section{DISCUSIÓN}

Vitamina D y sistema inmunitario. La vitamina D juega un papel esencial en el sistema inmune. La vitamina $\mathrm{D}$ interfiere con la mayoría de las células del sistema inmunitario, como los macrófagos, los linfocitos B y T, los neutrófilos y las células dendríticas, que expresan VDR. La catelicidina, un péptido formado por la expresión estimulada por la vitamina $D$, ha mostrado actividad antimicrobiana contra bacterias, hongos y virus envueltos, como los coronavirus. ${ }^{12}$ Además, la vitamina $\mathrm{D}$ inhibe la producción de citocinas proinflamatorias y aumenta la producción de citocinas antiinflamatorias. $^{13,14}$

\section{Definición del déficit de vitamina D}

En el año 2011 se publica la Guía de Práctica Clínica de la Sociedad de Endocrinología de Estados Unidos (EUA) sobre la evaluación, tratamiento y prevención de la deficiencia de vitamina D. En esta guía, se definen los distintos grados de déficit de vitamina $D$, y considera deficiencia un valor de $25 \mathrm{OHD}$ plasmática $\leq 20 \mathrm{ng} / \mathrm{mL}$; insuficiencia valores, entre 21 y $29 \mathrm{ng} / \mathrm{mL}$ y niveles óptimos, aquellos $\geq$ a $30 \mathrm{ng} / \mathrm{mL}$. Por otra parte, el Instituto de Medicina de EUA (IOM), basado en otros estudios en relación a niveles de PTH, sugiere que aproximadamente $97.5 \%$ de la población sana a través de diversos grupos etarios, alcanzan sus requerimientos de vitamina D con valores por sobre $20 \mathrm{ng} / \mathrm{mL}$, $^{5,10}$ Por lo tanto, no se ha establecido un consenso acerca del "umbral" para definir un estatus adecuado de vitamina D.

Estado de vitamina D En América Latina. La población de América Latina y el Caribe es de 583 millones de habitantes al año 2010 con diversos orígenes étnicos. La expectativa de vida promedio actual es de 74 años y se estima un crecimiento significativo de la población de personas de edad avanzada en la región. Los estudios de algunos países abordaron el estado de la vitamina $D$ en muestras pequeñas de poblaciones diversas. Es común la prevalencia de niveles bajos de vitamina $\mathrm{D}$ en los grupos de adultos mayores, sobre todo en los países de América Latina, especialmente en el grupo de mujeres adultas y adolescentes.

\section{Déficit de vitamina $D$ en profesionales de la salud}

Entre los grupos en riesgo de sufrir déficit de vitamina $D$, se encuentran los profesionales que pasan una mayor parte del día privados de exposición solar, con una consecuente disminución de su síntesis, siendo los profesionales de la salud un potencial grupo con mayor prevalencia a dicho déficit. Un estudio en el Hospital Pediátrico en Boston realiza mediciones de vitamina $\mathrm{D}$ junto a una encuesta alimentaria a un grupo de residentes de Pediatría a fines de invierno. De un total de 102 participantes, $25 \%$ tuvo niveles menores a $20 \mathrm{ng} / \mathrm{mL}$ y un $3 \%$ niveles menores a $10 \mathrm{ng} / \mathrm{mL}$. Como factores relacionados con niveles adecuados de vitamina $\mathrm{D}$ se encontraron: sexo femenino, raza blanca, viajar a latitudes ecuatoriales y alto consumo diario de suplementos. ${ }^{15-20}$ El metabolito activo de la vitamina $\mathrm{D}$ en los macrófagos y las células dendríticas, derivado del precursor $25(\mathrm{OH}) \mathrm{D}$, conduce a la activación de VDR que, después de la heterodimerización de receptor por retinoide (RXR), da como resultado la expresión de varias proteínas del sistema inmune innato y adaptativo ${ }^{22,23}$ (Células Tregs, citocinas, defensinas, receptores de reconocimiento de patrones, etcétera) y modulación de inmunoglobulinas. ${ }^{15,21,22}$

Otro estudio en México evalúa la prevalencia de déficit de vitamina $D$ e hiperparatiroidismo secundario en médicos residentes del Hospital de Porto Alegre. ${ }^{23,24}$ Se realizan mediciones a 73 médicos, con edad promedio de 26.4 años. Como resultado, se obtiene un nivel promedio de $25 \mathrm{OHD}$ de $17.9 \pm 8 \mathrm{ng} / \mathrm{mL}$, con $57.4 \%$ de individuos con niveles de deficiencia ( $\leq 20 \mathrm{ng} / \mathrm{mL}$ ). Como resultado, $25 \%$ de los participantes tuvo niveles deficientes $(\mathrm{mL})$ y $61.3 \%$ niveles insuficientes.

\section{CONCLUSIONES}

El déficit de vitamina $D$ parece ser un problema mundial en diferentes etapas de la vida, no sólo en los grupos de riesgo, por lo tanto, los estudios deben incluir diferentes poblaciones y estados fisiológicos.

Además, las comparaciones entre los países adquieren validez al utilizar un laboratorio central estandarizado para su medición.

Es necesario un consenso del mejor umbral para determinar qué pacientes con COVID-19 grave desarrollan una respuesta inflamatoria exagerada que finalmente pone en peligro su propia integridad. La vitamina $\mathrm{D}$ promueve el movimiento y la capacidad fagocítica en los macrófagos e induce la síntesis de sustancias químicas bactericidas como la catelicidina y la beta-defensina. Pero la vitamina $\mathrm{D}$ también modula 
la respuesta inflamatoria al reducir la expresión de citocinas proinflamatorias a través de la inducción de la diferenciación del macrófago M2 y la promoción de las células T-helper tipo 2 y T-reguladoras. Además, se ha demostrado que la vitamina $D$ regula el sistema renina-angiotensina y la expresión de ACE2 en modelos animales. El ACE2 es el receptor del huésped para el SARS-CoV-2 y está regulado negativamente por él. La desregulación del sistema renina-angiotensina aumenta la permeabilidad pulmonar y puede conducir al desarrollo de un síndrome respiratorio agudo severo. Además, los datos clínicos observacionales indican que la deficiencia de vitamina $\mathrm{D}(\mathrm{VDD})$ confiere un mayor riesgo de infecciones respiratorias y la suplementación con esta vitamina reduce este riesgo. En conjunto, todos estos datos proporcionan una justificación para considerar a la vitamina D como un factor importante implicado en la fisiopatología del SARS en COVID-19.

Es recomendable determinar el estatus de vitamina $\mathrm{D}$ basados en las implicaciones biológicas (óseas y extra óseas) de su déficit en los diferentes grupos, incluyendo su relación con la ingesta nutricional y los hábitos de exposición a luz ultravioleta.

\section{REFERENCIAS}

1. Norman AW. Sunlight, season, skin pigmentation, vitamin D, and 25-hydroxyvitamin D: integral components of the vitamin $D$ endocrine system. Am J Clin Nutr. 1998; 67 (6): 1108-1110.

2. Trincado MP. Hipovitaminosis D. Rev Med Clin Condes. 2013; 24 : 813-817.

3. Holick MF, Binkley NC, Bischoff-Ferrari HA, Gordon CM, Hanley DA, Heaney RP et al. Evaluation, treatment, and prevention of vitamin $D$ deficiency: an endocrine society clinical practice guideline. J Clin Endocrinol Metab. 2011; 96 (7): 1911-1930.

4. Vuolo L, Di Somma C, Faggiano A, Colao A. Vitamin D and cancer. Front Endocrinol. 2012; 3: 58.

5. Sun Q, Shi L, Rimm EB, Giovannucci EL, Hu FB, Manson JE et al. Vitamin $D$ intake and risk of cardiovascular disease in US men and women. Am J Clin Nutr. 2011; 94 (2): 534-542.

6. Chagas CE, Borges MC, Martini LA, Rogero MM. Focus on vitamin D, inflammation and type 2 diabetes. Nutrients. 2012; 4 (1): 52-67.

7. Holick MF. Vitamin D deficiency. N Engl J Med. 2007; 357 (3): 266281.

8. Hilger J, Friedel A, Herr R, Rausch T, Roos F, Wahl D et al. A systematic review of vitamin $\mathrm{D}$ status in populations worldwide. $\mathrm{Br}$ J Nutr. Br J Nutr. 2014; 111 (1): 23-45.
9. Morales-Torres J. Vitamin D deficiency across Latin America. Osteoporos Int. 2012; 23 (Suppl 4): S464-466.

10. Oliveri B, Plantalech L, Bagur A, Wittich AC, Rovai G, Pusiol E et al. High prevalence of vitamin $D$ Insufficiency in healthy elderly people living at home in Argentina. Eur J Clin Nutr. 2004; 58: 337-342.

11. Lips P, Hosking D, Lippuner K, Norquist JM, Wehren L, Maalouf $G$ et al. The prevalence of vitamin $D$ inadequacy amongst women with osteoporosis: an international epidemiological investigation. J Intern Med. 2006; 260: 245-254.

12. Saraiva GL, Cendoroglo MS, Ramos LR, Araújo LM, Vieira JG, Maeda SS et al. Prevalence of vitamin D deficiency, insufficiency and secondary hyperparathyroidism in the elderly in patients and living in the community of the city of Sao Paulo, Brazil. Arq Bras Endocrinol Metabol. 2007; 51: 437-442.

13. $Q u G, L i X$, Jiang $G$. An imperative need for research on the role of environmental factors in transmission of novel coronavirus (COVID-19). Environ Sci Technol. 2020; 12: 22-30.

14. Laird E, Rhodes J, Kenny RA. Vitamin D and inflammation: potential implications for severity of COVID-19. Ir Med J. 2020; 113: 81.

15. Sajadi MM, Habibzadeh P, Vintzileos A et al. Temperature, Humidity, and Latitude Analysis to Predict Potential Spread and Seasonality for COVID-19. 2020. SSRN Electronic Journal. Available in: https://doi.org/10.2139/ssrn.3550308

16. Price-Haywood EG, Burton J, Fort D, Seoane L. Hospitalization and mortality among black patients and white patients with COVID-19. N Engl J Med. 2020; 382: 2534-2543.

17. Kohlmeier M. Avoidance of vitamin D deficienc to slow the COVID-19 pandemic. BMJ Nutr Prev Health. 2020. Available in: https://doi.org/10.1136/bmjnph-2020-000096

18. Rhodes JM, Subramanian S, Laird E, Kenny RA. Editorial: low population mortality from COVID-19 in countries south of latitude 35 degrees North supports vitamin $\mathrm{D}$ as a factor determined severity. Aliment Pharmacol Ther [On Line). 2020; 51 (12): 14341437. Available in: https://doi.org/10.1111/apt.15777

19. Bouillon R, Marcocci C, Carmeliet G, Bikle D, White JH, DawsonHughes B et al. Skeletal and extraskeletal actions of vitamin D: current evidence and outstanding questions. Endocr Rev. 2019; 40: 1109-1151.

20. Prietl B, Treiber G, Piber TR, Amrein K. Vitamin D and immune function. Nutrients. 2013; 5: 2502-2521.

21. Di Rosa M, Malaguarnera M, Nicoletti F, Malaguarnera L. Vitamin D3: a helpful immune-modulator. Immunology. 2011; 134 (2): 123139.

22. Adams JS, Ren S, Liu PT, Chun RF, Lagishetty V, Gombart $\mathrm{AF}$ et al. Vitamin D-directed rheostatic regulation of monocyte antibacterial responses. J Immunol. 2009; 182 (7): 4289-4295.

23. Liu PT, Stenger S, Li H, Wenzel L, Tan BH, Krutzik SR et al. Toll-like receptor triggering of a vitamin $\mathrm{D}$ - mediated human antimicrobial response. Science. 2006; 311: 1770-1773.

24. Gombart AF, Pierre A, Maggini S. A review of micronutrients and the immune system-working in harmonyto reduce the risk of infection. Nutrients [On Line]. 2020; 12 (1): 236. Available in: https://doi.org/10.3390/nu12010236 